\title{
Improvement of the Intergranular Pinning Energy in the Na-doped Bi-2212
} Superconductors

\author{
$\underline{\text { B. Özçelik }}^{\mathrm{a}^{*}}, \underline{\text { M. Gürsul }}^{\mathrm{a}}, \underline{\text { A. Sotelo }}^{\mathrm{b}}, \underline{\text { M. A. Madre }}{ }^{\mathrm{b}}$ \\ ${ }^{a}$ Department of Physics, Faculty of Sciences\&Letters, Cukurova University, Adana, Turkey \\ bICMA (CSIC-Universidad de Zaragoza). María de Luna, 3. 50018 Zaragoza, Spain.
}

\begin{abstract}
In the present study, magnetoresistivity performance of polycrystalline $\mathrm{Bi}_{2} \mathrm{Sr}_{2} \mathrm{Ca}_{1-x} \mathrm{Na}_{x} \mathrm{Cu}_{2} \mathrm{O}_{8+y}$ superconductor with $x=0.0,0.05,0.075,0.10,0.15$, and 0.20 has been studied by change of flux pinning mechanism. Samples have been prepared using a polymer solution technique with polyethyleneimine. The effects of $\mathrm{Na}$ substitution for $\mathrm{Ca}$ on the activation energies, irreversibility field, upper critical magnetic field and coherence length have been studied. The magnetoresistance of samples has been measured at applied magnetic fields between 0 and 9 Tesla. Broadening of superconducting transition has been observed under magnetic field, explained on the basis of Thermally Activated Flux Flow (TAFF) model. The upper critical magnetic field $H_{C 2}(0)$ and the coherence length $(\zeta(0))$ at $T=0 \mathrm{~K}$ were calculated using the resistivity data and $H_{C 2}(0)$, respectively. $H_{C 2}(0)$ and $\xi(0)$ values have been calculated as 186.4 , 195.5, 321.0, 296.0, 292.5, 280.9 T, and 13.29, 12.98, 10.13, 10.55, 10.61, $10.69 \AA$ for the $0.0,0.05,0.075,0.10,0.15$, and $0.20 \mathrm{Na}$-doped samples, respectively. TAFF model has been studied in order to calculate the flux pinning energies. In particular, the flux pinning energies of $\mathrm{Bi}_{2} \mathrm{Sr}_{2} \mathrm{Ca}_{1-\mathrm{x}} \mathrm{Na}_{\mathrm{x}} \mathrm{Cu}_{2} \mathrm{O}_{8+\mathrm{y}}$ where $x=0.075$ determined to be $0.019 \mathrm{eV}$ for $9 \mathrm{~T}$ and $0.239 \mathrm{eV}$ for $0 \mathrm{~T}$.
\end{abstract}

Keywords: High $\mathrm{T}_{\mathrm{c}}$ superconductors, XRD, Magnetoresistance, Activation energy, TAFF, Upper critical magnetic field, coherence length

*Corresponding Author: Tel./fax:+90.322.3386060/2496/+90.322.3386070

e-mail: ozcelik@cu.edu.tr 


\section{INTRODUCTION}

Flux pinning is the phenomenon that magnetic flux lines do not move as they become trapped, or pinned in spite of the Lorentz force acting on them inside a current-carrying type-II superconductor. The motion of vortices in type-II superconductors and, in particular, in highTc superconducting (HTSC) cuprates is hindered by pinning forces associated with various structural defects. The efficient pinning centers are formed by extended growth defects, such as edge dislocations, twin planes, and grain boundaries as well as by artificial, radiationinduced columnar defects [1,2]. The nature and concentration of pinning sites in superconducting samples mainly depend on the grain boundaries or impurities resulting from the substitution into the matrix [1-4]. In order to prevent flux creep, which can create a pseudo-resistance and depress both critical current density and critical field in high temperature ceramic superconductors, flux pinning is a desirable phenomenon. Degradation of a high-temperature superconducting properties due to flux creep is a limiting factor constraining the use of HTc superconductors in technological applications.

Since Bi-based superconductors $\mathrm{Bi}_{2} \mathrm{Sr}_{2} \mathrm{Ca}_{n-1} \mathrm{Cu}_{n} \mathrm{O}_{x}(n=1,2,3)$ were discovered by Maeda et al. [5] many substitutions have been made in BSCCO system in order to improve their physical and magnetic properties [6-9]. In the last decades, the interest on BSCCO for both the fundamental research and applications in technology increases day by day owing to the fact that it is one of the most promising material for potential industrial applications such as superconducting wires, tapes, and thin films [10]. High- $T_{\mathrm{C}}$ Bi-based superconductor family is characterized by a high superconducting transition temperature $T_{\mathrm{C}} \sim 90$ and $110 \mathrm{~K}$ for the phases with $n=2$, and 3, respectively, and a high upper critical magnetic $B_{c 2}$ which can reach $150 \mathrm{~T}[11]$.

Due to their short coherence length, large anisotropy and thermal energy, it is rather difficult to realize the mixed state of high-temperature superconductors. For technological applications, the critical current density is a very important parameter of HTc superconductors in which the flux pinning mechanism determines the maximum current density driving through a superconductor without dissipation. Flux motion predicted by Anderson [12] is a mean of dissipating energy in superconductors. After this prediction of flux creep, large amounts of research have been addressed to determine the effect of applied external magnetic field on resistive transition in order to better understand the mechanism of flux pinning and flux motion [13-19]. On the other hand, as it is well-known, there are several models for understanding the resistivity broadening under a magnetic field; as thermally activated flux 
creep [15], flux flow [14], flux line melting and flux cutting [20], etc. The flux line can be thermally activated over the pinning energy barrier, even if the Lorentz force exerted on the flux bundle by the current is smaller than the pinning force. Some researchers have pointed out that a thermally activated flux creep model can describe quite well the data in the resistive region near $T_{\mathrm{C}}(\rho=0)[17, \underline{21-24]}$.

In addition, the material preparation technique plays a crucial role, as important as the substitutions, creating pinning centers in BSCCO systems. In order to easily obtain very homogeneous samples, the polymer matrix route is a very adequate solution methods, as reported in previous works [25-30]. In this method, smaller particle sizes than the produced by the classical solid state method, are is obtained leading to higher reactivity of powders and lower amount of secondary phases.

In previous works, the structural, physical and superconducting properties of Na-substituted BSCCO samples have been studied [31]. The present work aims: (i) introducing optimum amounts of $\mathrm{Na}$ into the $\mathrm{Bi}_{2} \mathrm{Sr}_{2} \mathrm{Ca}_{1-\mathrm{x}} \mathrm{Na}_{\mathrm{x}} \mathrm{Cu}_{2} \mathrm{O}_{8+\mathrm{y}}$ system by replacing $\mathrm{Ca}$ due to their similar ionic radii but different oxidation state. Ca shows an oxidation state +2 while $\mathrm{Na}$ is +1 . As a consequence, modifications can be produced in the crystal structure, together with changes in carrier concentration due to changes in oxygen content. (ii) exploring the magnetoresisitivity, irreversibility field, upper critical magnetic field, coherence length and applying the thermally activated flux creep model on the resistivity data obtained under various applied magnetic fields between 0-9 T, calculating the activation energies of these modified systems.

\section{EXPERIMENTAL DETAILS}

$\mathrm{Bi}_{2} \mathrm{Sr}_{2} \mathrm{Ca}_{1-\mathrm{x}} \mathrm{Na}_{\mathrm{x}} \mathrm{Cu}_{2} \mathrm{O}_{\mathrm{y}}$ samples, with $\mathrm{x}=0,0.05,0.075,0.1,0.15$, and 0.20 have been prepared by using a polymer matrix route described in detail elsewhere [25,32]. $\mathrm{Bi}\left(\mathrm{CH}_{3} \mathrm{COO}\right)_{3}$ ( $\geq 99.99 \%$, Aldrich), $\mathrm{Sr}\left(\mathrm{CH}_{3} \mathrm{COO}\right)_{2} \bullet 0.5 \mathrm{H}_{2} \mathrm{O}\left(99 \%\right.$, Panreac), $\mathrm{Ca}\left(\mathrm{CH}_{3} \mathrm{COO}\right)_{2} \cdot 2 \mathrm{H}_{2} \mathrm{O}(98 \%$, Alfa Aesar), $\mathrm{Cu}\left(\mathrm{CH}_{3} \mathrm{COO}\right)_{2} \bullet \mathrm{H}_{2} \mathrm{O}$ (98\%, Panreac) and $\mathrm{Na}\left(\mathrm{CH}_{3} \mathrm{COO}\right)$ (99\%, Alfa Aesar) commercial powders were used as starting materials. They were weighed in stoichiometric amounts and dissolved in a mixture of glacial acetic acid $\left(\mathrm{CH}_{3} \mathrm{COOH}\right)$ (Panreac PA) and distilled water. The use of a mixture of glacial acetic acid and water is due to the fact that in one side $\mathrm{Sr}$ acetate is insoluble in concentrated acetic acid and, on the other side, Bi acetate is not soluble in water [33]. Once a clear blue solution is obtained, polyethyleneimine (PEI) (Aldrich, 50 wt.\% water) was added. The mixture becomes dark blue immediately reflecting the formation of $\mathrm{Cu}-\mathrm{N}$ coordination bonds. The solution was then introduced into a rotary 
evaporator to reduce its volume (in $\sim 80 \%$ ), followed by heating on a hot plate at about $100{ }^{\circ} \mathrm{C}$ for total solvent evaporation, producing a thermoplastic dark blue paste. Further heating at around $350{ }^{\circ} \mathrm{C}$ produces a decomposition step (self-combustion), as described schematically elsewhere [37], which produces the organic material decomposition. The resulting powder was then milled in an agate mortar and calcined twice at 750 and $800^{\circ} \mathrm{C}$ for $12 \mathrm{~h}$, with an intermediate manual milling, in order to decompose the alkaline-earth carbonates.

Finally, the prereacted homogeneous powders were pressed into $13 \mathrm{~mm}$ diameter pellets, and thermally treated in order to produce the Bi-2212 superconducting phase. This process was performed under air, and consisted in two steps: $60 \mathrm{~h}$ at $860{ }^{\circ} \mathrm{C}$, followed by $12 \mathrm{~h}$ at $800{ }^{\circ} \mathrm{C}$ and, finally, quenched in air to room temperature.

In order to identify the present phases, powder X-ray diffraction patterns of the materials were recorded at room temperature using a Rigaku D/max-B powder diffractometer system working with $\mathrm{CuK} \alpha$ radiation and a constant scan rate between $2 \theta=3-60^{\circ}$. The uncertainty of the crystal lattice parameters calculation remained in the \pm 0.00001 range. Magnetoresistivity measurements were carried out in a Quantum Design PPMS system. The $\mathrm{Bi}_{2} \mathrm{Sr}_{2} \mathrm{Ca}_{1}$ ${ }_{x} \mathrm{Na}_{\mathrm{x}} \mathrm{Cu}_{2} \mathrm{O}_{8+\mathrm{y}}$ samples, with $\mathrm{x}=0,0.05,0.075,0.1,0.15$, and 0.20 will be hereafter named as $\mathrm{Na} 01, \mathrm{Na} 02, \mathrm{Na} 03, \mathrm{Na} 04, \mathrm{Na} 05$, and $\mathrm{Na} 06$, respectively.

\section{RESULTS AND DISCUSSION}

\subsection{XRD characterization}

XRD patterns of all samples are exhibited in Fig.1. The peaks corresponding to the Bi2212 and $\mathrm{CaCuO}_{2}$ phases are indicated by + and $*$, respectively. It is clear that all samples are mainly composed by the Bi-2212 phase, independently of the $\mathrm{Na}$ amount. The crystal symmetry of all samples was determined to be tetragonal and the calculated lattice parameters using the least squares method are presented in Table 1. As it can be inferred from these data, while $c$-parameter is decreasing, $a-b$ parameters are slightly increasing when Na-content is increased. These variations are due to the fact that the insertion of $\mathrm{Na}^{1+}$ substituting $\mathrm{Ca}^{2+}$ ions in the structure produces a decrease on the global positive charge in the structure. As a consequence, a modification on the oxygen content has to be produced to maintain the electrical neutrality in the structure, decreasing $c$-parameter and, consequently, raising the $a-b$. Additionally, it is found that no changes in crystal symmetry with variation of $\mathrm{Na}$ concentration have been observed. 
In order to get more information about the crystal sizes, Debye Scherrer formula [34] has been applied to the XRD data, as:

$$
L_{h k l}=0.9 \lambda / \beta \cos \theta
$$

where $\lambda$ is the used wavelength, $\beta$ is the full width at half maximum of X-ray peaks and $\theta$ is the angle of the peak. As can be seen from Table 1, the particle sizes increase with increasing Na-concentration, probably due to the decrease of the system melting point induced by $\mathrm{Na}$. As a consequence, the growth process speed is increased due to the formation of a small amount of liquid phase.

\subsection{Charge Carrier Concentration}

In order to understand the mechanism of the doping dependence of transition temperature, $T c$, in the HTc superconductors, the calculation of the charge carrier concentration, $p$, in the $\mathrm{CuO}_{2}$ planes is of fundamental interest. Presland et al. [35] suggested the empirical relation;

$$
T_{c} / T_{c}^{\max }=1-82.6(p-0.16)^{2}
$$

between $T_{c}$ and Sr-hole doping in $\mathrm{La}_{1-\mathrm{x}} \mathrm{Sr}_{\mathrm{x}} \mathrm{CuO}_{4}$, where $T_{c}^{\max }$ is the maximum attainable $T_{c}$, and $p$ is the hole concentration per $\mathrm{Cu}$ atom in the $\mathrm{CuO}_{2}$ planes. According to Eq.2, $T_{c}$ is maximized for hole concentrations $\approx 0.16$ and falls to zero at $\approx 0.05$ on the underdoped side and at $\approx 0.27$ on the overdoped side. The above relation has been found to be a remarkable model to determine the $T_{c}$ values versus doping concentration [36]. Therefore, the relation has often been used as a convenient representation of the relation between $T_{c}$ and the hole concentration for all the hole doped cuprates. The calculated $p$ values for the samples are displayed in Table 2. These results show that the $p$-values are very close to each other. However, these values are close to the obtained in the undoped Bi-2223 phase. These results indicate that the amount of $\mathrm{Na}$ dopant is far from the overdoping limit, in agreement with the results of Satyavathi et al. [37].

\subsection{Thermoelectric Power}

The thermoelectric power, $S$, is highly sensitive to the charge transport mechanism. Hence, it gives information related to the nature of the charge carriers, carrier concentration and band structure. The studies of $S$ as a function of carrier concentration and temperature have been done in the Bi-based system [38]. It has been established that the high- $T_{c}$ superconducting oxides are strongly correlated systems. The expression of $S$, at high 
temperatures was derived by different groups $[39,40]$, and was modified by Cooper et al. [41]; according to these studies, the thermoelectric power of HTc materials at high temperatures can be described by the formula;

$$
\left.\left.S=k_{B} / e[\ln (1-p) / 2 p)-\ln 2\right)\right]
$$

where $p, k_{B}$ and $e$ are the hole concentration, Boltzmann constant and electron charge, respectively. Using the $p$-values given in Table 2, the $S$ values for the samples have been calculated and tabulated Table 2. As it can be seen, all the values are negative, clearly indicating a dominating electron conduction mechanism.

\subsection{Magnetoresistivity measurements}

In order to investigate the effect of Na-doping on the superconducting properties of the samples, the magnetoresistivity experiments have been performed on the samples in the range of $150 \mathrm{~K}$ down to $20 \mathrm{~K}$, for various magnetic fields between 0 and $9 \mathrm{~T}$. In Figure-2, the field dependent resistivity of the samples as a function of temperature is presented. The onset $\left(T_{c}^{\text {onset }}\right)$ and offset $\left(T_{c}^{\text {offset }}\right)$ temperatures concerning the transition of isolated grains to the superconducting state and to the volume fraction of Bi-2212 phase and/or characteristic of intergranular component, respectively, are summarized in Table 3. The $T_{c}^{\text {onset }}$ values have been taken from the crossing point of the linear fit of the highest slope and the metallic high temperature part of the $\rho(T)$ curve for each sample. Since the onset transition temperature $\left(T_{c}{ }^{\text {onset }}\right.$ ) is above $90 \mathrm{~K}$, it can be argued that the high- $T c$ Bi-2212 phase is the dominant in the samples. It can be seen that at zero magnetic field both $T_{c}^{\text {onset }}$ and $T_{c}^{\text {offset }}$ values increase from 91.268 $\mathrm{K}$ to $93.863 \mathrm{~K}$, and from $80.033 \mathrm{~K}$ to $81.763 \mathrm{~K}$, respectively, with raising Na-content in the samples. This increase in the temperature values is originated due to the increment in the number of charge carriers in the $\mathrm{CuO}_{2}$ planes.

The slight shifts towards high temperatures with increasing magnetic field observed in the $T_{c}^{\text {onset }}$ may be probably associated with the raise of the superconducting fraction together with the carrier concentrations. Also, this behavior could be attributed to the strong intragrain pinning energy not allowing any vortex motion in the vicinity of $T c$ [17]. On the other hand, while the temperature lowers, the electrical resistivity becomes more sensitive to the applied magnetic field and the resistivity tail appears at lower temperatures. This is a clear indication that at lower temperatures more charge carriers become superconducting, so the change between the resistivity measured at zero and the applied field becomes greater [42], and strong Josephson type links concerning the grain-boundaries. 


\subsection{Activation Energy}

In order to investigate in detail the effect of $\mathrm{Na}$ substitution on the superconducting properties, it is possible to deduce some physical parameters as activation energy, irreversibility field and coherence length. The study of thermally activated flux flow (TAFF) is manifested as a broadening of the superconducting transition. The resistivity in the TAFF region is caused by the thermally activated flux flow of the vortices. The experimental results in the tail part have been found to follow the Arrhenius relation given in [43]

$$
\rho(H, T)=\rho_{0} \exp \left(-U(H) / k_{B} T\right)
$$

where $U$ is the flux pinning energy that depends on temperature and magnetic field. The $U$ value can be directly deduced from the slope of the plot of $\ln \left(\rho / \rho_{0}\right)$ versus $1 / T$. Fig. 3 shows the Arrhenius plots for all the samples. From these figures, it is obvious that the resistive broadening caused by an applied magnetic field is reflected in a $T_{c}$ shift to lower temperatures. This is a well-known behavior attributed to the flux flow resistivity. Since it is well established, lowering the temperature increases the pinning strength hence the pinning force. The other result which can be deduced from the semi-logarithmic Arrhenius plots of Fig. 3, is that indeed there is nearly an exponential dependence of $\rho$ on $1 / T$ for $T<<T_{c}^{\text {onset }}$, indicating the energy dissipation in the TAFF region due to the thermal activation of fluxes across the pinning barriers. Therefore, $U$ values are obtained from the slopes of the nearly straight line portions of the curves at low temperatures.

In Fig.4, the calculated activation energy, $U$, from the linear fit in the tail part of the plots is exhibited, for all samples. As it can be seen in all cases, the activation energy decreases with the increase of applied magnetic field. It is evident from this figure that the activation energies of the samples increase significantly with the enhancement in both the $\mathrm{Na}$ doping level and applied magnetic field. It is possible to say that the raise of the energy barriers may arise from the increasing Na-content which cause the increasing of the pinning centers, by substituting Ca. Since these pinning centers strongly behave as effective pinning centers in the matrix, the applied fields do not significantly penetrate into the sample producing an increase of critical current values. In this case, the high critical current density values are the evidence of the strong pinning mechanism arising from Na-substitution. In our previous study, it has been obtained that the critical currents were increased with the Nacontent up to $\mathrm{x}=0.10$ [31]. This effect can reveal an increase of the intergranular coupling and a reduction of the amount of weak links. With a decrease of the weak links, the vortices do not creep easily due to the relatively large pinning energy or activation energy values. It will 
be also worthwhile to mention that the main differences between curves in Figure 4 can be observed for fields below 3 Tesla. Since below 3 Tesla, the applied magnetic field has only penetrated the intergranular region, a possible interpretation for the differences between curves can be ascribed to the existence of different superconducting levels within the samples in these regions: the superconducting grains, the weak-links, and the superconducting clusters [44-46]. Thus, it would be reasonable to assure that in sample Na03, the fraction of grains interconnected by strong-links (clusters) is very large as compared with those of the other samples.

\subsection{Irreversibility Line, Irreversibility Field and Coherence Length}

The irreversibility line (IL) is one of the basic properties of HTSC. It fundamentally displays the change from the flux pinning dominated regime to that dominated by the thermally activated flux flow (TAFF), where resistance appears. The irreversibility field, $H_{i r r}$, has been defined from [48] and plotted in Figure 5. Plotting the irreversibility limits $H_{\text {irr }}$ of the samples for various applied fields in an $H$ - $T$ diagram generates an IL that separates the $H$ - $T$ plane into two regions: a low temperature one, in which the flux dynamics is dissipative and thus irreversible, and a high temperature one, where the flux motion is free and magnetically reversible. As seen in Figure 5, $H_{\text {irr }}$ increases and IL shifts to higher temperatures with increasing Na-content, then it decreases. These results indicate that the strength vortex fluctuations are getting stronger with increasing $\mathrm{Na}$-content.

In order to determine the behavior of the upper critical field at temperatures close to zero, a theoretical model, defined by Werthamer-Helfand-Hohenberg (WHH) has been used, applying the formula [60];

$$
\mu_{0} H_{c 2}(0)=0.693 T_{c}\left(d \mu_{0} H_{c 2}(T) / d T\right)
$$

where the $\left(d H_{c 2}(T) / d T\right)$ are determined using $T_{C}$ values determined from the midpoint of the linear fit of the highest slope of the resistivity curves at zero field given in Figure 2.

Another important parameter in the superconductivity is the coherence length, $\xi$. In the framework of this study, the coherence length, $\xi$, has been calculated using the GinzburgLandau relation,

$$
\mu_{0} H_{c 2}(0)=\Phi_{0} / 2 \pi \xi^{2}(0)
$$

where $\Phi_{0}$ is the quanta of flux $\left(2.07 \times 10^{-15} \mathrm{~T}^{2} \mathrm{~m}^{2}\right)$ and $H_{c 2}(0)$ is the upper critical field at zero temperature. The calculated upper critical field, $H_{c 2}$ and the coherence length, $\xi$, versus the critical temperatures are given in Table 2. The calculated upper critical fields and the 
coherence length at zero $\mathrm{K}$ for $\mathrm{H} / \mathrm{c}$ are 321.0 Tesla and $10.13 \AA$ for sample $\mathrm{Na} 03$, respectively.

\section{CONCLUSIONS}

In summary, polycrystalline $\mathrm{Bi}_{2} \mathrm{Sr}_{2} \mathrm{Ca}_{1-x} \mathrm{Na}_{x} \mathrm{Cu}_{2} \mathrm{O}_{8+y}$ superconductors with $x=0.0,0.05,0.075$, $0.10,0.15$, and 0.20 have been prepared by the polymer matrix method. From XRD patterns, the crystal symmetry of all samples is determined to be tetragonal. The calculated lattice parameters are found while $c$-parameter is decreasing, $a-b$ parameters are slightly increasing, when Na-content is increased. The calculated charge carrier concentration $(p)$ values are very similar in all cases. The calculated thermoelectric power $(S)$ values are found as negative, indicating a dominating electron conduction mechanism. The change of the flux pinning mechanism has been studied using magnetoresistivity measurements. The activation energies, irreversibility fields, upper critical fields and coherence lengths at $0 \mathrm{~K}$ were evaluated from the resistivity versus temperature $(\rho-T)$ curves, under DC magnetic fields up to $9 \mathrm{~T}$. The electrical resistivity broadening behavior in the magnetic field has been studied using the thermally activated flux creep model. According to this model, initial substitution of $\mathrm{Na}$ up to $x=0.075$ increases the flux pinning energy, $U$, which indicates higher slope in the energy barriers. This is an evidence of the increase of the energy barriers and pinning centers arising from $\mathrm{Na}$ addition. These pinning centers strongly act as effective pinning centers in the matrix. Hence, the applied fields do not significantly penetrate into the sample producing an increase of critical current values. This can be attributed to an increase of the intergranular coupling and a reduction of the amount of weak links. With a decrease of the weak links, the vortices do not creep easily due to the relatively large values pinning energy or activation energy.

\section{Acknowledgement}

This work is supported by Research Fund of Cukurova University, Adana, Turkey, under grant contracts no: FEF 2013YL18 and FEF2013BAP22. 


\section{References:}

[1] J. M. Huijbregtse, B. Dam, R. C. F. van der Geest, F. C. Klaassen, R. Elberse, J. H.Rector, and R. Griessen, Phys. Rev. B 62, 1338 (2000).

[2] J. Albrecht, Phys. Rev. B 68, 054508 (2003).

[3] M. Hawley, I. D. Raistrick, J. G. Beery, and R. J. Houlton, Science 251, 1587 (1991).

[4] C. Gerber, D. Anselmetti, J. G. Bednorz, J. Mannhart, and D. G. Schlom, Nature (London) 350, 279 (1991).

[5] H.Maeda, Y. Tanaka, M. Fukutomi, T. Asano, Jpn. J. Appl. Phys. 27, L 209 (1988)

[6] B.Chattopadhyay, B.Bandyopadhyay, A.Poddar, P.Mandal, A.N.Das, B.Ghosh, Physica C $331,38(2000)$

[7] G.Ilonca, V.Toma, T.R.Yang, A.V.Pop, P.Balint, M. Bodea, E.Macocian, Physica C 369, 460 (2007)

[8] X.L.Wang, J.Horvat, G.D.Gu, K.K.Uprety, H.K.Liu, S.X.Dou, Physica C 337, 221 (2000)

[9] T.Yamamoto, I.Kakeya, K.Kadowaki, Physica C 460-462, 799 (2007)

[10] M. Cyrot, D. Pavuna, Introduction to Superconductivity and High-Tc Materials, p. 249. World Scientific, Singapore (1995)

[11] G. Blatter, M.V. Feigelman, V.B. Ceshkenbein, A.I. Larkin, V.M. Vinokur, Rev. Mod. Phys. 6, 1125 (1994)

[12] P.W. Anderson, Phys.Rev.Lett. 9,309 (1962)

[13] M.Erdem, O.Ozturk, E.Yucel, S.P.Altintas, A.Varilci, C.Terzioğlu, I.Belenli, Physica B 406, 705 (2011)

[14] M.Tinkham, Phys. Rev. Lett. 61, 1658 (1988)

[15] T.T.Palstra, B.Batlogg, R.B. Van Dover, L.F. Scheemeyer, J. V.Waszczak, Appl. Phys. Lett. 54, 763 (1989)

[16] D.H. Kim, K. F. Gray, R. T. Kampwirth, D. M. Mckay: Phys, Rev. B 42,6249 (1990)

[17] B.Özkurt, B.Özçelik, J. Low Temp.Phys. 156, 22 (2009)

[18] D.Yazici, M.Erdem, B.Özçelik, J. Supercond. Nov.Magn. 25, 1811 (2012)

[19] D. Sharma, R. Kumar, V.P.S. Awana, Solid State Commun. 152, 941 (2012)

[20] M.R.Mohammadizadeh, M.Akvahan, Physica C 390, 134 (2003)

[21] T.T. Palstra, B. Batlogg, L.F. Schneemeyer, J.V. Waszczak, Phys. Rev. Lett. 61 1662 (1988).

[22] A.P. Malozemoff, T.K. Worthington, E. Zeldov, N.C. Yeh, M.W. McElfresh, in Strong Correlation and Superconductivity, ed. by H. Fukuyama, S. Maekawa, A.P. Malozemoff. 
Springer Series in Sol.State Sci., vol. 89, Springer, Berlin, (1989)

[23] R.Griessen, Phys. Rev. Lett. 64, 1674 (1990)

[24] R.C. Ma, W.H. Song, X.B. Zhu, L. Zhang, S.M. Liu, J. Fang, J.J. Du, Y.P. Sun, C.S. Li, Z.M. Yu, Y. Feng, P.X. Zhang., Physica C 405, 34 (2004)

[25] G. F. de la Fuente, A. Sotelo, Y. Huang, M. T. Ruiz, A. Badia, L. A. Angurel, F. Lera, R. Navarro, C. Rillo, R. Ibañez, D. Beltran, F. Sapiña, A. Beltran, Physica C, 185, 509 (1991)

[26] B. Özçelik, B. Özkurt, M.E. Yakınc1, A. Sotelo, M. A. Madre, J. Supercond. Nov. Magn., 26, 873 (2013)

[27] A.Sotelo, H.Szillat, P. Majewski, F. Aldinger, Supercond. Sci. Technol. 10, 717-720 (1997)

[28] H. Gündogmus, B. Özçelik, B. Özkurt, A. Sotelo, M.A. Madre, J. Supercond. Nov. Magn, 26, 111(2013)

[29] A. Ozaslan, B. Özçelik, B. Özkurt, A. Sotelo, M.A. Madre, J. Supercond. Nov. Magn, 27, 53 (2014)

[30] C. Kaya, B. Özçelik, B. Özkurt, A. Sotelo, M.A. Madre, J Mater Sci:Mater Electron, 24, $1580(2013)$

[31] B. Ozcelik, M. Gursul, A. Sotelo, M. A. Madre, J Mater Sci: Mater Electron.(in press)

\section{DOI 10.1007/s10854-014-2190-x}

[32] A. Sotelo, Sh. Rasekh, M.A. Madre, J.C. Diez: J. Supercond. Nov. Magn. 24, 19 (2011)

[33] D.R. Lide: CRC Handbook of Chemistry and Physics. CRC Press/Taylor and Francis, Boca Raton, FL (2010)

[34] B. D. Cullity, Element of X-ray Differaction, Addition-Wesley, Reading, MA (1978)

[35] M.R.Presland, J.L. Tallon, R.G. Buckley, R.S. Liv, N.E. Floer, PhysicaC, 176, 95 (1991)

[36] J.B. Torrance, A. Bezinge, A.I. Nazzal, Phys. Rev. B 40, 8872 (1989)

[37] S. Satyavathi, K. Nanda Kishore, V. Hari Babu, O. Pena, Supercond. Sci. Technol., 9, 93, (1996)

[38] M. C. Sekhar and S.V. Suryanarayana, Physica C, 415, 209 (2004)

[39] G. Beni, Phys. Rev. B10, 2187 (1974)

[40] P.M.Chaikin, G.Beni, Phys. Rev.B 46, 647 (1976)

[41] J.R. Cooper, B. Alavi, L.W. Zhow, W.P. Boyermann, G. Gruner, Phys. Rev. B 35, 8794 (1987)

[42] N.G. Gomaa, Egypt. J. Solid 22, 256, (1999)

[43] J. J. Kim, H. Lee, J. Chung, H.J. Shin, H. J. Lee, J.K. Ku, Phys. Rev. B, 43, 2962 (1991) 
[44] E.Govea-Alcaide,I.Garcia-Fornaris, P.Mune, and R.F.Jardim, Eur.Phys.J.B 58, 373, (2007)

[45] P. Kameli, H. Salamati, I. Abdolhosseini, D. Sohrabi, Physica C 468, 137, (2008)

[46] D. Yazıcı, B. Özçelik, M. E. Yakıncı, J Low Temp Phys 163, 370, (2011)

[47] N.R. Werthamer, E. Helfand, P.C. Hohenberg, Phys. Rev. 147, 295 (1966)

[48] H. Gündogmus, B. Özçelik, A. Sotelo, M.A. Madre, J Mater Sci: Mater Electron 24, $2568(2013)$ 
Table.1. Unit cell parameters and crystal size for each sample

\begin{tabular}{|c|c|c|c|}
\hline Samples & $\begin{array}{l}\text { Unit-cell } \\
\text { parameter } \\
a=b(\AA)\end{array}$ & $\begin{array}{l}\text { Unit-cell } \\
\text { parameter } \\
c(\AA)\end{array}$ & $\boldsymbol{L}_{\mathbf{h k l}}(\AA)$ \\
\hline $\mathbf{N a 0 1}$ & 5.4280 & 30.7678 & 424.74 \\
\hline $\mathbf{N a 0 2}$ & 5.4283 & 30.7530 & 432.68 \\
\hline $\mathbf{N a 0 3}$ & 5.4336 & 30.7330 & 445.44 \\
\hline $\mathbf{N a 0 4}$ & 5.4391 & 30.7322 & 446.85 \\
\hline $\mathbf{N a 0 5}$ & 5.4415 & 30.7318 & 450.45 \\
\hline $\mathbf{N a 0 6}$ & 5.4494 & 30.7290 & 463.68 \\
\hline
\end{tabular}


Table.2. Physical properties of all the samples

\begin{tabular}{|c|c|c|c|c|}
\hline Sample & $P$ & $S\left(10^{-4} V / K\right)$ & $H_{c 2}(0)(T)$ & $\xi\left(A^{0}\right)$ \\
\hline $\mathrm{Na01}$ & 0.1825 & -7.610 & 186.4 & 13.29 \\
\hline $\mathrm{NaO2}$ & 0.1811 & -7.026 & 195.5 & 12.98 \\
\hline $\mathrm{NaO3}$ & 0.1786 & -6.173 & 321.0 & 10.13 \\
\hline $\mathrm{Na04}$ & 0.1807 & -6.874 & 296.0 & 10.55 \\
\hline $\mathrm{Na05}$ & 0.1787 & -6.204 & 292.5 & 10.61 \\
\hline $\mathrm{NaO6}$ & 0.1730 & -6.834 & 280.9 & 10.69 \\
\hline
\end{tabular}


Table.3. $T_{c}^{\text {onset }}$ and $T_{c}^{\text {offset }}$ values of the samples at different applied magnetic fields

\begin{tabular}{|c|c|c|c|c|c|c|}
\hline Sample & 0 Tesla & 1 Tesla & 3 Tesla & 5 Tesla & 7 Tesla & 9 Tesla \\
\hline \multirow{2}{*}{$\begin{array}{ll}N a 01 & T_{c}^{\text {onset }} \\
& T_{c}^{\text {offset }}\end{array}$} & 91.268 & 90.879 & 90.326 & 89.497 & 89.065 & 88.515 \\
\hline & 80.033 & 4.361 & 54.500 & 47.704 & 44.041 & 41.290 \\
\hline \multirow{2}{*}{$\begin{array}{ll}\text { Na02 } & T_{c}^{\text {onset }} \\
& T_{c}^{\text {offset }}\end{array}$} & & & 90.411 & & 89.194 & 88.621 \\
\hline & 81.487 & 65.057 & 55.243 & 49.903 & 46.488 & 43.845 \\
\hline \multirow{2}{*}{$\begin{array}{ll}\text { Na03 } & T_{c}^{\text {onset }} \\
& T_{c}^{\text {offset }}\end{array}$} & 92.780 & 92.457 & 92.205 & 91.925 & 91.476 & 91.062 \\
\hline & 80.585 & 68.096 & 59.739 & 54.351 & 51.434 & 48.695 \\
\hline \multirow{2}{*}{$\begin{array}{ll}\mathrm{Na04} & \mathrm{T}_{c}^{\text {onset }} \\
& \mathrm{T}_{c}^{\text {offset }}\end{array}$} & 91.618 & 91.287 & 90.931 & 90.594 & 90.120 & 89.842 \\
\hline & 77.953 & 62.761 & 53.738 & 48.520 & 45.278 & 42.643 \\
\hline \multirow{2}{*}{$\begin{array}{ll}\text { Na05 } & T_{c}^{\text {onset }} \\
& T_{c}^{\text {offset }}\end{array}$} & 92.457 & 92.194 & 91.855 & 91.481 & 90.979 & 90.653 \\
\hline & 79.507 & 63.393 & 54.286 & 49.064 & 46.111 & 43.692 \\
\hline \multirow{2}{*}{$\begin{array}{ll}\text { Na06 } & T_{c}^{\text {onset }} \\
& T_{c}^{\text {offset }}\end{array}$} & 93.863 & 93.599 & 93.125 & 92.982 & 92.643 & 92.283 \\
\hline & 81.763 & 66.903 & 58.192 & 52.102 & 49.104 & 46.909 \\
\hline
\end{tabular}




\section{FIGURE CAPTIONS}

Figure 1. XRD patterns of samples. In figure, a-f indicates the samples Na01-Na06, respectively. Peaks corresponding to the $\mathrm{Bi}-2212$ and $\mathrm{CaCuO}_{2}$ phases are indicated by + and *, respectively.

Figure 2. Temperature dependence of resistivity for various applied magnetic fields for all the samples.

Figure 3. Arrhenius plot of the resistivity of all the samples

Figure 4. Flux pinning energy $U$ versus applied magnetic field for all the samples

Figure 5. Irreversibility field $\left(H_{i r r}\right)$ versus temperature 
Figure 1

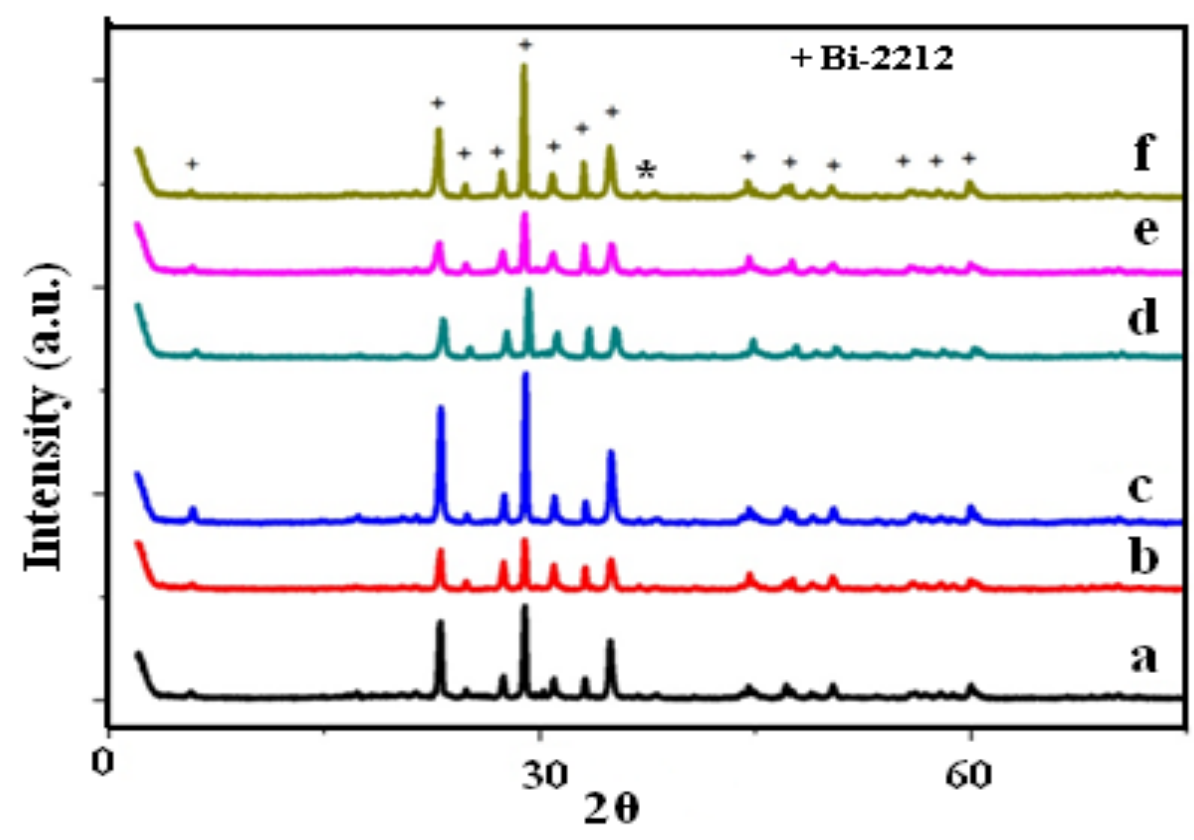


Figure 2
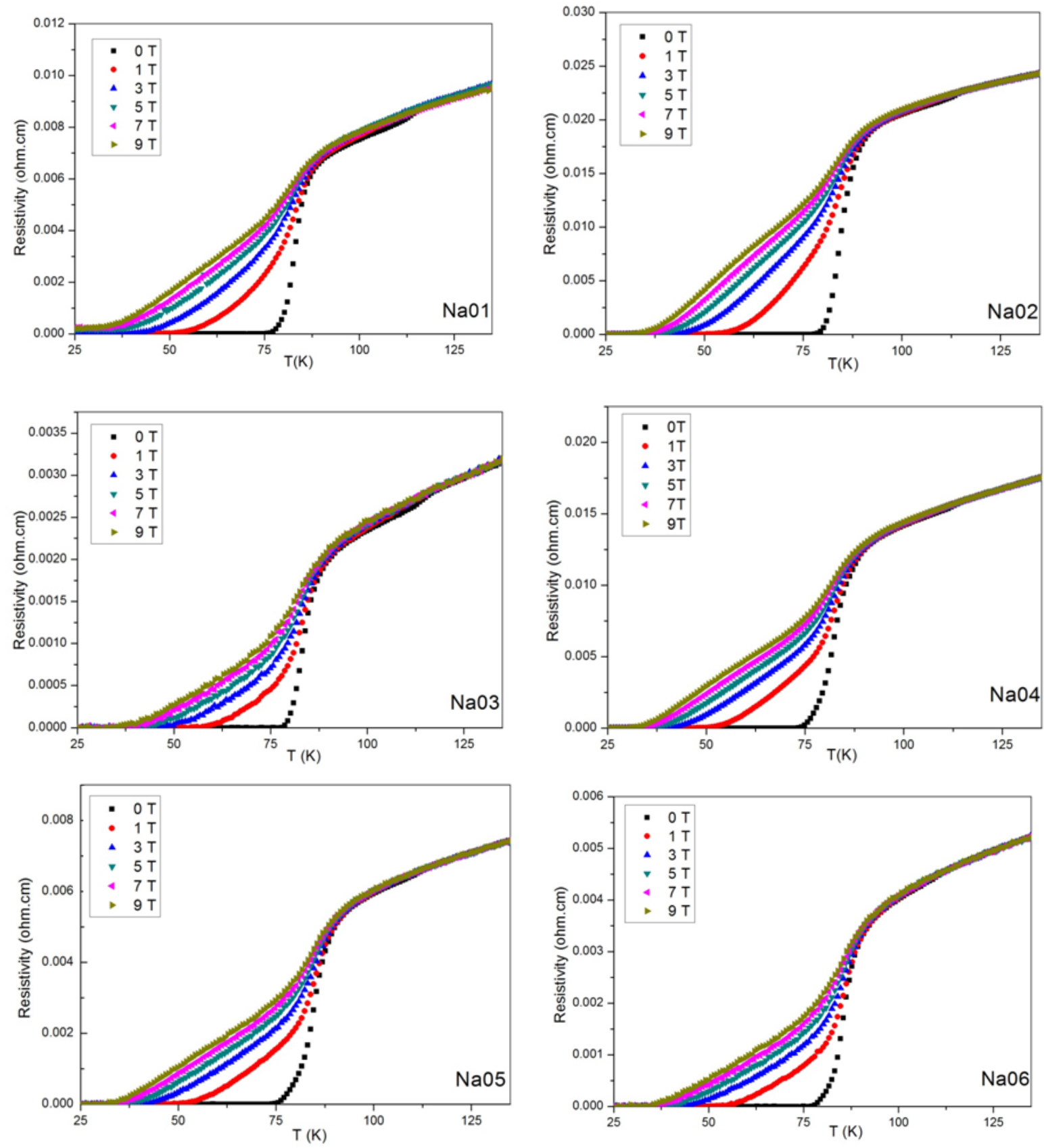
Figure 3
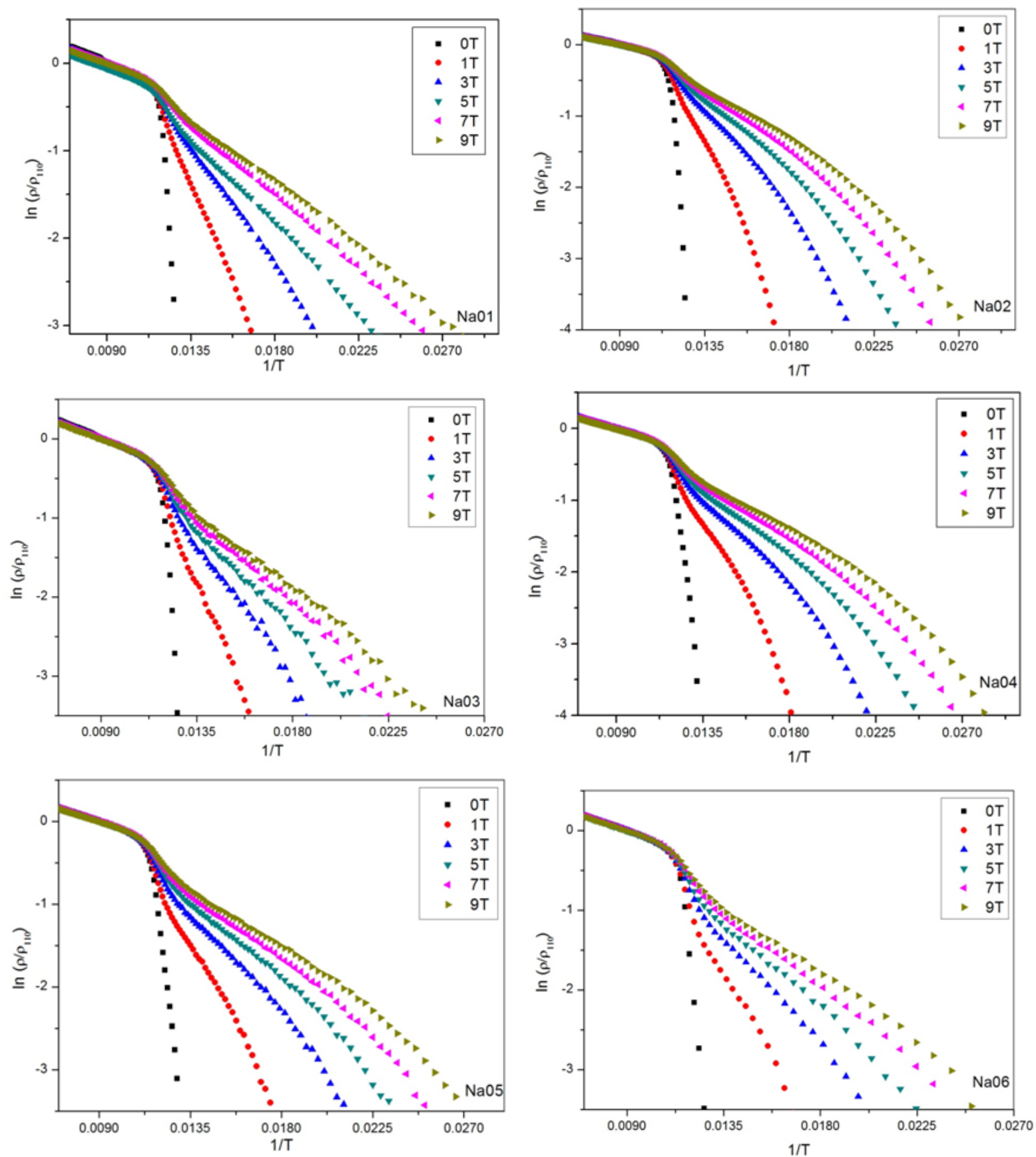
Figure 4

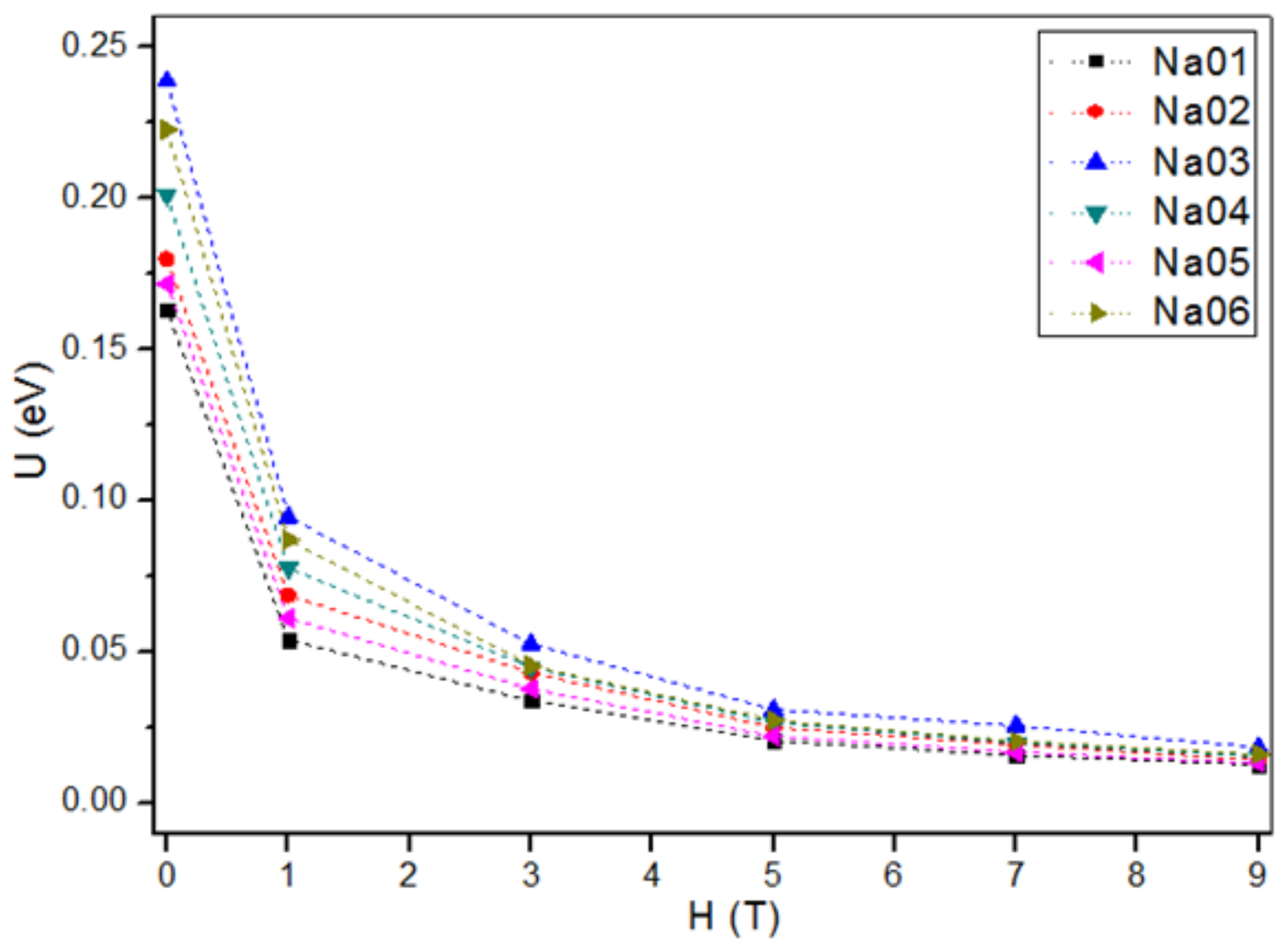


Figure 5

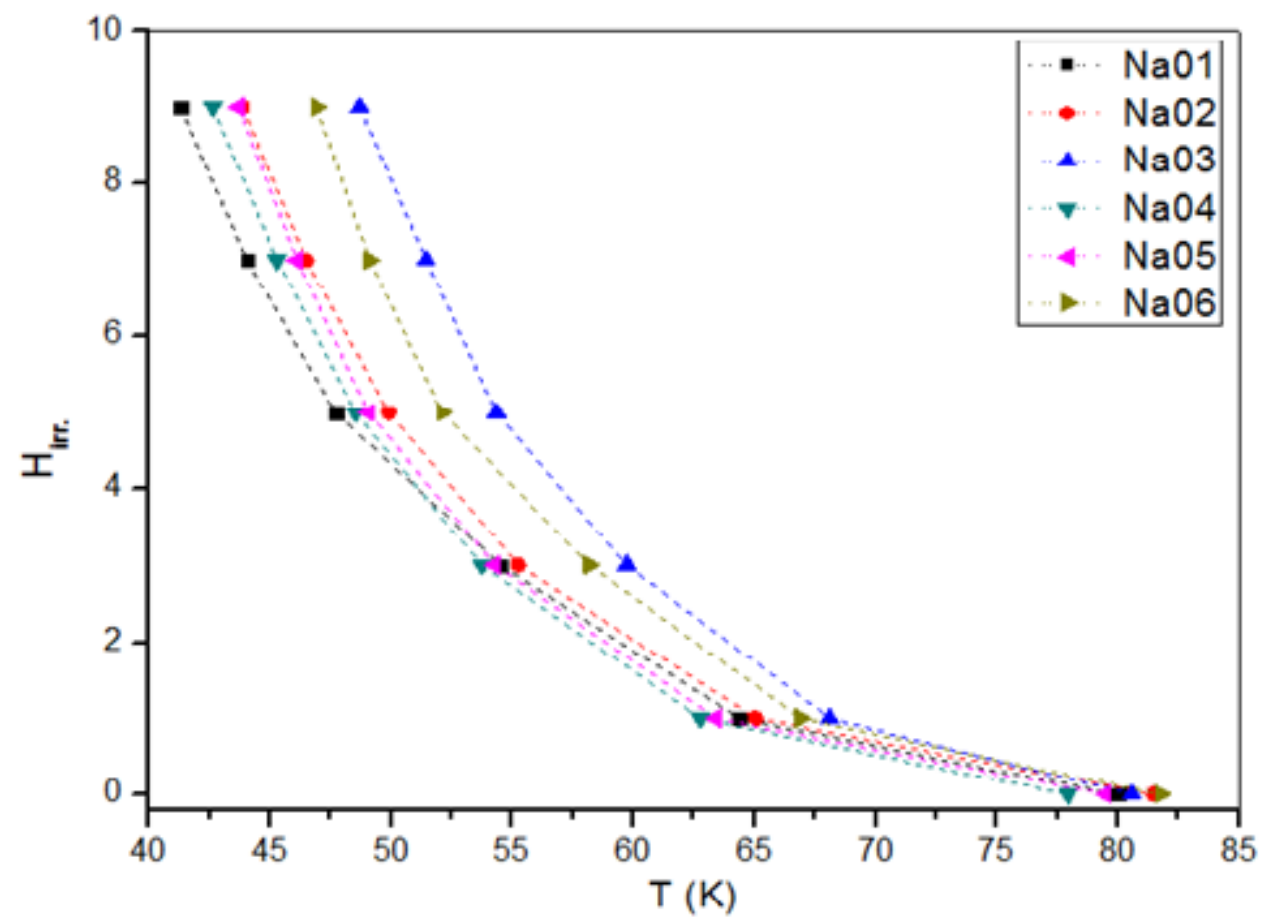

